\title{
CHANGES OF LISM CHARACTERISTICS IN THE HELIOSPHERIC INTERFACE
}

\author{
Hartmut W. Ripken and Hans J. Fahr \\ Institut für Astrophysik, Universität Bonn, F.R. Germany
}

\section{ABSTRACT}

It is possible to deduce LISM properties from observations of interste1lar neutral gases in the inner solar system. Parameters accessible by this method are the interstellar wind vector and the densities and temperatures of hydrogen and helium, implying also the deduction of the relative abundance ratios and the degree of ionization in the LISM. Direct inference from observations, for example resonance luminescence measurements of Jy-alpha and He-58.4 $\mathrm{nm}$ radiation, yields values appropriate only for the inner solar system, i.e. for the regions within the heliopause dominated by the solar wind plasma.

Particularly the subsonic LISM plasma interface ahead of the heliopause causes profound changes in the properties of the neutral LISM gas traversing this region. Mainly $\mathrm{p}-\mathrm{H}$ charge exchange processes give rise to the destruction of primary hydrogen and the production of secondary hydrogen atoms, the net effect being a depletion of the neutral hydrogen component of the LISM by about $50 \%$.

Details on the depletion mechanisms, the hydrogen and oxygen extinctions, and the consequences for the Ly-alpha resonance luminescence intensity interpretations are presented.

\section{INTRODUCTION}

Neutrals penetrating the region of a perturbed interstellar plasma flow along individual dynamical trajectories cannot be treated as moving under conservation of total energy and angular momentum, since their collisional mean free paths in most cases are smaller than the interface dimensions. Due to large charge exchange cross sections, especially $\mathrm{H}$ and 0 atoms effectively undergo charge exchange collisions with interstellar protons. This leads to both destruction of one sort of neutrals and production of another sort of neutrals with different dynamical trajectories. Thus the flow of neutrals along a specific trajectory s through the interface is not carried exclusively by those primary particles that have originally entered the perturbed region from the unperturbed one. Instead, primary particles are effectively removed from the trajectory $s$ via charge exchange processes, and neutrals belonging to other trajectories undergo charge exchange reactions with jons moving along $s$ at the instant of interaction, thus causing a repopulation of the trajectory $s$. In this respect, the primary neutrals on $s$ are gradually, and in an extended subsonic interface entirely, replaced by secondary particles.

\section{THE BOLTZMANN FORMULATION}

The local change of the velocity distribution function $f$ of the neutrals is thus defined by the local balance between destruction and production rates. This is adequately formulated in the characteristics form of 
Boltzmann's integro-differential equation for charge exchange collisions (see Ripken and Fahr (1983), Eqs. (2), (3), and (5)),

$$
\begin{aligned}
v \frac{d}{d s}\left(f_{n}\right) & =f_{i}(\underline{r}, \underline{v}) \rho^{3} f_{n}\left(\underline{r}, \underline{v}_{n}\right) v_{r e 1}\left(\underline{v}, \underline{v}_{n}\right) \sigma_{e x}\left(v_{r e 1}\right) d^{3} \underline{v}_{n}- \\
& -f_{n}(\underline{r}, \underline{v}) f^{3} f_{i}\left(\underline{r}_{v} \underline{v}_{i}\right) v_{r e 1}\left(\underline{v}, \underline{v}_{i}\right) \sigma_{e x}\left(v_{r e 1}\right) d^{3} \underline{v}_{i},
\end{aligned}
$$

where $f_{i}$ is the ion velocity distribution function, $v_{\text {rel }}$ is the relative velocity between the collision partners, and $\sigma$ is the velocity-dependent charge exchange cross section. The differential line element ds is measured along the specific dynamical trajectory $s$ that belongs to the particles with a velocity $\underline{v}$ at a place $\underline{r}$. The integrations are carried out over the entire velocity space. It needs to be stressed that in this form Eq. (1) fully incorporates the multiple collision concept and thus represents a complete treatment of the underlying redistribution effects.

The relative importanceof loss and gain terms critically depends on the type of species treated, the relevant individual particle velocity $\underline{v}$ along $s$, and the current position $\underline{r}$ in the interface. Due to the very small cross sections for charge exchange collisions between $\mathrm{He}$ atoms and protons (2 $10^{-17} \mathrm{~cm}^{-2}$ ), charge exchange interactions of interstellar helium atoms in the pre-heliospheric plasma interface can be neglected. Other helium losses due to electron impact ionizations and critical velocity effect ionizations (Petelski et al., 1980) can be disregarded also since sufficient ionization energies are not available.

This is different for $O$ and $H$ atoms that traverse the interface. In these cases the relevant cross sections for chafge exchange $\left(\mathrm{O}-\mathrm{H}^{+}, \mathrm{H}-\mathrm{H}^{+}\right)$are much larger (i.e. of the order of $10^{-15} \mathrm{~cm}^{2}$ ). For 0 atoms the charge exchange collisions with protons only represent a loss process, whereas 0 atom gains only result from charge exchange collisions between $0^{f}$ interfaceions and $\mathrm{H}$ or $\mathrm{O}$ atoms. Assuming a cosmic abundance of 0 atoms and a degree of ionization comparable to that of interstellar hydrogen, $\left(\mathrm{N}_{\mathrm{p}} / \mathrm{N}_{\mathrm{H}}\right)_{\infty}$ ح $\left(\mathrm{N}_{\mathrm{O}_{+}} / \mathrm{N}_{\mathrm{O}}\right)_{\infty}$, and realizing that production processes are predominantly proportional to $\mathrm{N}_{\mathrm{l}}$, one can only expect loss processes for 0 atoms. Therefore the depletion of 0 atoms during the traversal of the heliospheric interface region is much larger than that of $H$ atoms. The different elemental depletions can qualitatively be extracted from $F$ ig. 1 ( 0 atom depletion: corresponding to curve $\mathrm{B}, \mathrm{H}$ atom depletion: curve $\mathrm{A}$ ). In addition, at any given specific position in the 0 atom velocity space the local production and loss rates are greatly disbalanced, thus causing pronounced changes in the strongly depleted 0 atom velocity distribution function $f_{0}$.

Changes of the $\mathrm{H}$ atom velocity distribution function $f_{H}$ ăre predominantly caused by charge exchange collisions between $\mathrm{H}$ atoms $\mathrm{H}^{\mathrm{H}}$ an protons. The resulting changes of $f_{H}$ lead to a $\mathrm{H}$ atom depletion factor of 0.45 . Thus, for boundary values of $\mathrm{N}_{\mathrm{p}_{2}}=0.0135 \mathrm{~cm}^{-3}$ and $\mathrm{N}_{\mathrm{H}^{\infty}}=0.1105 \mathrm{~cm}^{-3}$, at the solar wind shock $\mathrm{N}_{\mathrm{H}}=0.05 \mathrm{~cm}^{-3}$ is realized, with a corresponding hydrogen temperature increase of $10 \%$.

\section{PRODUCTION AND LOSS PROCESSES}

Under thermodynamic equilibrium conditions a detailed balance exists between collisional losses and gains for each cell in velocity space; i.e. 


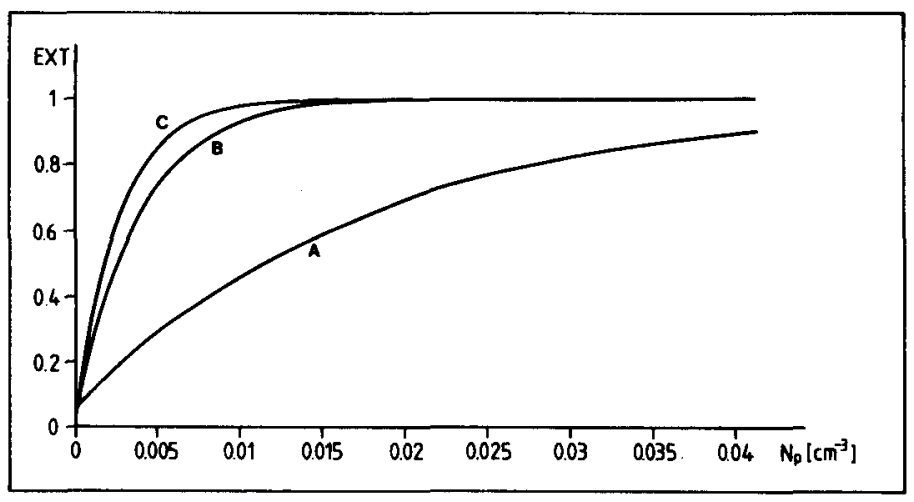

Figure 1: Hydrogen extinction EXT as a function of the asymptotic LISM proton density $\mathrm{N}_{\infty}$ Curve $\mathrm{A}$ : calculated including Both production and loss terms; curve $\mathrm{B}$ : calculated using only loss terms, with $\sigma_{\text {ex }}=\sigma_{\text {ex }}\left(v_{\text {rel }}\right) ; \quad$ curve $\sigma_{\text {same }}=610^{-1 \dot{5}}$ $\mathrm{cm}^{2}$

$\mathrm{df} / \mathrm{ds}=0$. Such an equilibrium state can be adopted between ions and neutrals in the unperturbed interstellar medium far ahead of the heliosphere. However, in the region where the interstellar plasma flow approaches the heliosphere and becomes hydrodynamically perturbed and deflected around the impermeable heliospheric obstacle, a non-equilibrium between neutrals and ions is built up because the dynamical coupling of the two species is only very weak. The ion distribution function $f_{i}$, due to the action of elfetromagnetic forces and plasma waves, drastically changes over the last $10^{2}$ to $10^{3} \mathrm{AU}$ ahead of the heliosphere, whereas the neutral distribution function cannot fully adapt to these rapidly varying ion conditions and attains an intermediate state.

A note concerning the balance of $\mathrm{H}$ atom production and loss terms and the method of calculation is in order here. For an extended subsonic interface like that proposed by Parker (1963) a solution of the form

$f_{n}(s)=f_{n}\left(s_{\infty}\right) \exp \left(-\int_{s_{\infty}}^{s} \frac{v_{r e 1}}{v} \sigma_{\text {ex }} N_{p} d s\right)$

obtained from Eq. (1) when neglecting the production term is entirely unrealistic since no reasonable upper limit for $s_{\infty}$ can be defined. Thus for $\mathbf{s}_{\infty} \rightarrow_{\infty}$ the function $\mathrm{f}_{\mathrm{n}}$ and thus also $\mathrm{N}_{\mathrm{H}}$ would systematically tend towards zero. This is due to ${ }^{n}$ the fact that even in the unperturbed plasma regions the loss processes do not drop to zero. Nevertheless, in the unperturbed region these loss processes are completely compensated by gain processes, and $f$ remains unchanged.

The importance of treating the production term in Eq. (1) properly is further emphasized when considering Fig. 1. Curve B shows the extinction

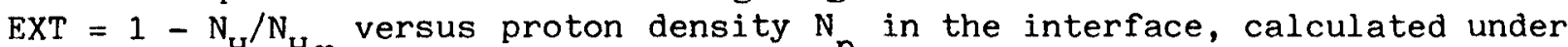
neglect of the production term (i.e. using a formula of the type $\mathrm{N} \sim 1-\exp (-\tau))$. This yields absolutely unrealistic values of the extinction. In contrast, inclusion of particle gain processes leads to the realistic curve A. This curve is not derivable under "forward scattering" or "destruction" concepts employing depletion factor expressions of $\left(1+\langle 1-x>\tau)^{-1}\right.$ (Wallis, 1984) or $\exp (-\tau)$.

An interesting consequence of the interface-related $\mathrm{H}$-atoms depletion is a revised form of how the unperturbed interstellar hydrogen densities $\mathrm{N}_{\mathrm{H}}$ correlate with the interplanetary Ly-alpha resonance luminescence intensities. In the earlier theory, reviewed for instance by Thomas (1978), a 


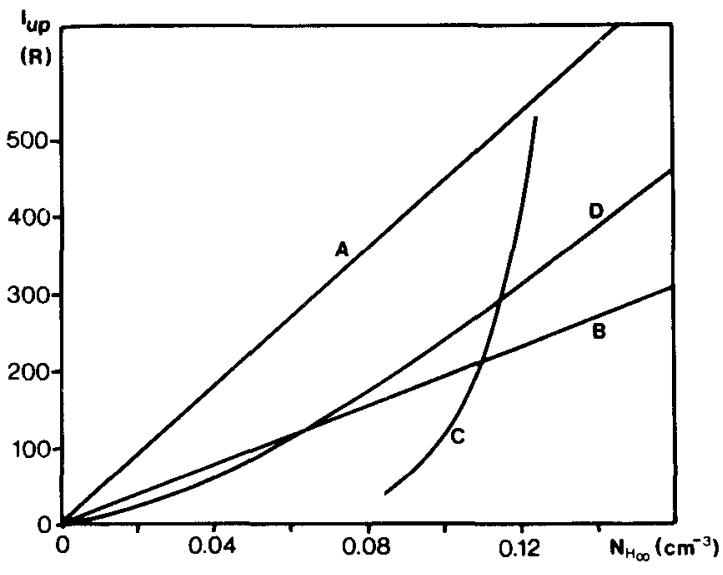

Figure 2: Upwind Ly-alpha resonance luminescence intensities versus asymptotic hydrogen density $\mathrm{N}_{\mathrm{H}^{\circ}}$. Curve A: "Classical" dependence without interface extinction; curve $B$ : with interface extinction, and constant $\mathrm{N}_{\text {poo }}$; curve $C$ : with interface extinction, using $\left(\mathrm{N}_{\mathrm{H}^{\circ}}+\mathrm{N}_{\mathrm{p}}\right)$ = const; curve $\mathrm{D}$ : selfconsistent ${ }^{\mathrm{H} \infty}$ interface of Baranov (1981), using only loss processes. Curves $A, B$, and $C$ use as a reference point the interplanetary Ly-alpha observations of Bertaux et al. (1976).

linear relation between the upwind Ly-alpha intensity $I_{\text {up }}$ and the unperturbed hydrogen density $\mathrm{N}_{H^{\infty}}$ was expected (curve $\mathrm{A}$ in $\mathrm{Fig} . \mathrm{up}_{2}$ ). In the model of Ripken and Fahr (1983), a strongly varying nonlinear relation is found (curve C) due to the boundary value $\left(\mathrm{N}_{\mathrm{H}^{\infty}}+\mathrm{N}_{\mathrm{p}^{\infty}}\right)=10 \mathrm{~N}_{\mathrm{He}}$ employed in the model. Baranov (1981), though only treating Poss processes, has calculated the resulting interface configuration in the two-shock approach self-consistently. In this case a different nonlinear relation of $I_{u p}$ and $N_{H o o}$, shown in
curve $D$, was obtained.

A rigorous treatment of both loss and gain processes, leading to the "luminosity-hydrogen density" relationship characterized by curve C, suggests that observed upwind Ly-alpha intensities strongly depend on the actual interstellar hydrogen density $\mathrm{N}_{\mathrm{H}^{\infty}}$, and that the possible range of the densities $\mathrm{N}_{\mathrm{H}^{\infty}}$ is narrowed down considerably as compared to the conventional solution in curve $A$. On the other hand, due to the steepness of curve $C$, an observational inaccuracy in $I_{\text {up }}$ (e.g. by about $\pm 50 \mathrm{R}$ ) only leads to small differences in the derived interstellar hydrogen densities $\mathrm{N}_{\mathrm{H} \infty}$ (e.g. by about $\left.\pm 0.002 \mathrm{~cm}^{-3}\right)$.

\section{REFERENCES}

Baranov, V.B.: 1981, private communication

Bertaux, J.L., J.E. Blamont, N. Tabarie, W.G. Kurt, M.C. Bourgin, A.S. Smi.rnov, and N.N. Dementeva: 1976, Astron. Astrophys. 46, 19-22

Parker, E.N.: 1963, Interplanetary Dynamical Processes, Interscience, New York

Petelski, E.F., Fahr, H.J., Ripken, H.W., Brenning, N., Axnäs, I.: 1980, Astron. Astrophys. 87, 20

Ripken, H.W., and Fahr, H.J.: 1983, Astron. Astrophys. 122, 181

Thomas, G.E.: 1978, Ann. Rev. Farth Planetary Sci. 6, 173

Wall is, M.K.: 1984, Astron. Astrophys. 130, 200 